\title{
REPRESENTATION OF TREE PERMUTATIONS BY WORDS
}

\author{
JOHN A. MAROLI
}

(Communicated by Louis J. Ratliff, Jr.)

\begin{abstract}
The problem of solving equations in groups can be stated as follows: given a group $G$ and a free group $F=F\left(x_{1}, x_{2}, \ldots\right)$, for which pairs $(w, g)$ with $w=w\left(x_{1}, x_{2}, \ldots\right) \in F, g \in G$, is it possible to find elements $g_{i} \in G$ such that $w\left(g_{1}, g_{2}, \ldots\right)=g$ ? We investigate the corresponding question of solving equations in the group $A(\Omega)$ of all automorphisms of a transitive tree $\Omega$. If the tree has isomorphic cones at a branch point, then certain equations of the form $x^{n}=g$ cannot be solved (Theorem 2.3). If the tree is sufficiently transitive, we find large classes of equations $w=g$ which can be solved (Theorems $2.13,2.16)$.
\end{abstract}

\section{INTRODUCTION}

A p.o. set $(\Omega, \leq)$ is called a tree or lower semilinear order if the following three conditions are satisfied:

(a) for each $\alpha \in \Omega$ the set $\{\tau \in \Omega: \tau \leq \alpha\}$ is totally ordered (a chain);

(b) for all $\alpha, \beta \in \Omega$ there is $\tau \in \Omega$ with $\tau \leq \alpha$ and $\tau \leq \beta$;

(c) $(\Omega, \leq)$ contains an infinite subchain.

A tree which is not a chain will be called a strict tree. A tree permutation group is a pair $(G, \Omega)$, where $\Omega$ is a tree and $G$ is a subgroup of the orderautomorphism group $A(\Omega)$; i.e., $g \in A(\Omega)$ if and only if $g$ is a one-to-one function from $\Omega$ onto $\Omega$ that satisfies for $\alpha, \beta \in \Omega, \alpha g \leq \beta g$ if and only if $\alpha \leq \beta$. Let $(G, \Omega)$ be a tree permutation group. If $\Omega$ is a chain, we say that $(G, \Omega)$ is an ordered permutation group and we endow $G$ with the pointwise ordering. $f \leq g$ if $\alpha f \leq \alpha g$ for all $\alpha \in \Omega$. If $(G, \Omega)$ is an ordered permutation group, and for each $f, g \in G$, the elements $f \vee g$ and $f \wedge g$ of $A(\Omega)$ actually belong to $G$, we say that $(G, \Omega)$ is an $\ell$-permutation group.

If $G$ is a permutation group and $g$ is an odd permutation, the equation $x^{2}=$ $g$ has no solution. Lyndon [6] and Mycielski [9] recently settled a conjecture of Silberger [10] by showing that if $G$ is the full symmetric group on an infinite set, and if $w$ is not a power (greater than 1) of any element of $F$, then the equation $w=g$ can always be solved in $G$. Adeleke and Holland [1] have recently proved results for the ordered analogue, i.e., when $G$ is the automorphism

Received by the editors July 1, 1989 and, in revised form, February 16, 1990.

1980 Mathematics Subject Classification (1985 Revision). Primary 06A10; Secondary 20B27. 
group of a transitive chain. In this paper we consider the more general case when $G$ is the order-automorphism group of a transitive tree, and we extend many of the results of Adeleke and Holland [1].

Throughout this paper we will use $\mathbf{Z}$ for the integers, and $\mathbf{R}$ for the reals. $\mathbf{Z}^{+}$will denote the positive integers, and $\mathbf{Z}^{-}$will denote the set of negative integers. Unless otherwise specified, these sets will all possess their natural total order. For any set $K,|K|$ will denote the cardinality of $K$, and $S(K)$ will denote the full symmetric group on $K$. We denote by $e$ the identity element of any group.

\section{BACKGROUND AND NOTATION}

For elements $\alpha, \beta$ of a partially ordered set $\Omega$, if either $\alpha \leq \beta$ or $\beta<\alpha$ we say that $\alpha$ and $\beta$ are comparable, otherwise we say that $\alpha$ and $\beta$ are incomparable and write $\alpha \| \beta$. If $\alpha \leq \beta$ we write $[\alpha, \beta]=\{\tau \in \Omega: \alpha \leq \tau \leq \beta\}$ for the closed interval, and $(\alpha, \beta)=\{\tau \in \Omega: \alpha<\tau<\beta\}$ for the open interval. A subset $S$ of $\Omega$ is said to be convex in $\Omega$ if whenever $\alpha, \beta \in S$ and $\alpha \leq \tau \leq \beta(\tau \in \Omega)$, then $\tau \in S$.

We say that a tree permutation group $(G, \Omega)$ is $k$-homogeneous if every isomorphism between $k$-element subsets of $\Omega$ extends to an element of $G$; $k$-transitive if, whenever two $k$-element subsets of $\Omega$ are isomorphic, there is an element of $G$ taking one subset to the other; weakly $k$-transitive if any isomorphism between subchains of $\Omega$ of length $k$ extends to an element of $G$. Note that weak $k$-transitivity is weaker than $k$-transitivity which is weaker then $k$-homogeneity. However, they all coincide if $\Omega$ is a chain or $k=1$. If $(G, \Omega)$ is 1 -transitive, we say that $(G, \Omega)$ is transitive. We also say that $\Omega$ is weakly $k$-transitive ( $k$-transitive, $k$-homogeneous) if $A(\Omega)$ is $\left(k \in \mathbf{Z}^{+}\right)$.

A partially ordered set $(P, \leq)$ is called Dedekind-complete if each nonempty subset of $P$ which is bounded below in $P$ has an infimum (greatest lower bound) in $P$, or equivalently, if each nonempty subset of $P$ which is bounded above in $P$ has a supremum (least upper bound) in $P$. It is easy to see that a tree $(\Omega, \leq)$ is Dedekind-complete if and only if each maximal subchain of $\Omega$ is Dedekind-complete and $\Omega$ is a meet-semilattice. The following proposition shows that each tree $(\Omega, \leq)$ has a unique Dedekind-completion.

Proposition 1.1. Let $(\Omega, \leq)$ be a tree. Then there is a Dedekind-complete tree $\left(\bar{\Omega}, \leq^{\prime}\right)$ with the following properties:

(i) $\Omega \subseteq \bar{\Omega}$, and $\leq, \leq$ coincide on $\Omega$;

(ii) if $\alpha \in \bar{\Omega}$, then there is a nonempty subset $S$ of $\Omega$, with $S$ bounded below in $\Omega$, such that $\alpha$ is equal to the greatest lower bound of $S$ in $\bar{\Omega}$.

Further, given trees $\Omega_{1}, \Omega_{2}$ and corresponding Dedekind-complete trees $\bar{\Omega}_{1}, \bar{\Omega}_{2}$ satisfying (i) and (ii), each isomorphism $\Phi: \Omega_{1} \rightarrow \Omega_{2}$ extends to a unique isomorphism $\bar{\Phi}: \bar{\Omega}_{1} \rightarrow \bar{\Omega}_{2}$. 
Proof. Call a convex subchain $C \subseteq \Omega$, with $C$ unbounded below in $\Omega$ and $C$ not a maximal subchain of $\Omega$, good if $C=L(U(C))$ where, for any subset $S$ of $\Omega, L(S)=\{\alpha \in \Omega$ : for all $\tau \in S, \alpha \leq \tau\}$ and $U(S)=\{\alpha \in \Omega$ : for all $\tau \in$ $S, \tau \leq \alpha\}$. For each $\alpha \in \Omega$, the set $C_{\alpha}=\{\tau \in \Omega: \tau \leq \alpha\}$ is a good chain. Let $\left(\bar{\Omega}, \leq^{\prime}\right)$ be the set of all good chains in $\Omega$, partially ordered by inclusion. Now embed $\Omega$ into $\bar{\Omega}$ via the map $\alpha \rightarrow C_{\alpha}(\alpha \in \Omega)$ and identify $\Omega$ with its image in $\bar{\Omega}$. Conditions (i) and (ii) follow, and $\left(\bar{\Omega}, \leq^{\prime}\right)$ is a Dedekind-complete tree. Finally, if $\Omega_{1}$ and $\Omega_{2}$ are trees and $\Phi: \Omega_{1} \rightarrow \Omega_{2}$ is an isomorphism, the mapping $\bar{\Phi}: \bar{\Omega}_{1} \rightarrow \bar{\Omega}_{2}$ defined by $\alpha \bar{\Phi}=\sup \left\{\tau \Phi: \tau \in \Omega_{1}, \tau \in \alpha\right\}$ is an isomorphism extending $\Phi$; clearly $\bar{\Phi}$ is unique.

We shall denote by $\bar{\Omega}$ the Dedekind-completion of a tree $\Omega$. Note that each $g \in A(\Omega)$ extends to a unique automorphism of $\bar{\Omega}$, which we will also denote by $g$. An element of $\bar{\Omega} \backslash \Omega$ will be called a hole of $\Omega$.

If $A$ is a subset (usually a chain) of a tree $\Omega$, we define $A^{c l}$ (the closure of $A$ in $\Omega$ ) by $A^{c l}:=\{\tau \in \Omega$ : there are $\alpha, \beta \in A$ such that either $\alpha \leq \tau \leq \beta$, or $\alpha<\tau$ and $\tau \| \beta\}$. If $\alpha, \beta \in \Omega$ with $\alpha \leq \beta$, we define $\langle\alpha, \beta\rangle:=\{\alpha, \beta\}^{c l}$ and call $\langle\alpha, \beta\rangle$ an interval of $\Omega$. If $\Omega$ has no maximal elements, $\alpha$ will be the smallest element of $\langle\alpha, \beta\rangle$ and $\beta$ will be the unique maximal element of $\langle\alpha, \beta\rangle$, and hence any isomorphism from $\langle\alpha, \beta\rangle$ to $\langle\tau, \sigma\rangle$ will take $\alpha$ to $\tau$ and $\beta$ to $\sigma$.

Recall the terminology from Droste, Holland, and Macpherson [3]. A convex subset $S$ of a tree $\Omega$ is said to be nontrivial orbital of $g \in A(\Omega)$ if $S$ is an infinite chain, $S g=S$, and for some (equivalently, all) $\alpha \in S$ the set $\left\{\alpha g^{i}: i \in \mathbf{Z}\right\}$ is unbounded above and below in $S$. We say that $g$ has positive (negative) parity on $S$ if $\alpha<\alpha g(\alpha g<\alpha)$ for some (equivalently, all) $\alpha \in S$.

A subgroup $G$ of $A(\Omega)$ is said to be depressible if, whenever $g \in G, S$ a nontrivial orbital of $g$, and $f \in A(\Omega)$ coincides with $g$ on $S^{c l}$ and fixes $\Omega \backslash S^{c l}$ pointwise, we have $f \in G$. Clearly, for any tree $\Omega, A(\Omega)$ is depressible.

Next, let $(G, \Omega)$ be a tree permutation group and $C \subseteq \Omega$ a convex subchain. We define $G_{C}:=\{g \in G: C g=C\}$, and $\left.G\right|_{C}:=G_{C} / N$, where $N$ is the normal subgroup $\left\{g \in G_{C}:\left.g\right|_{C}=e\right\}$ of $G_{C}$. If $C=\{\alpha\}$, we will write $G_{\alpha}$ for $G_{\{\alpha\}}$. Note that $\left.G\right|_{C}$ may be considered as a subgroup of $A(C)$. Let $g \in G$; the support of $g$, denoted by $\operatorname{supp}(g)$, is the set $\{\alpha \in \Omega: \alpha g \neq \alpha\}$.

A transitive ordered permutation group $(G, \Omega)$ is said to be primitive if it has no nontrivial congruences, where a congruence of $(G, \Omega)$ is an equivalence relation on $\Omega$ with convex classes which are preserved by each $g \in G$. An ordered permutation group $(G, \Omega)$ is said to be uniquely transitive if given $\alpha, \beta \in \Omega$, there is a unique $g \in G$ such that $\alpha g=\beta$. Our work is dependent upon the following classification due to McCleary [8].

Theorem 1.2. Let $(G, \Omega)$ be a transitive primitive depressible $/$-permutation group. Then either $(G, \Omega)$ is doubly transitive, or $(G, \Omega)$ is the right regular representation of a subgroup of $\mathbf{R}$ and thus uniquely transitive. 


\section{REPRESENTATION OF TREE PERMUTATIONS BY WORDS}

We say that a word $w$ in a free group $F$ is universal for a group $G$ if for every $g \in G$, the equation $w=g$ can be solved in $G$. Following Adeleke and Holland [1], we now give an example.

Consider $G=A(\mathbf{Z})$. Now $A(\mathbf{Z})$ is isomorphic to $\mathbf{Z}$, and hence, abelian. Let $t_{n}$ denote translation of $\mathbf{Z}$ by the integer $n$. If $w^{\prime}=\prod x_{i}^{m_{i}}$ is the abelianized form of $w$ (e.g., if $w=x^{2} y^{2} x^{-1} y$, then $w^{\prime}=x y^{3}$ ) and $w^{\prime}$ is a power, that is, g.c.d. $\left(m_{i}\right) \neq 1$, then clearly the equation $w=t_{1}$ cannot be solved in $A(\mathbf{Z})$, so $w$ is not universal for $A(\mathbf{Z})$. On the other hand, if g.c.d. $\left(m_{i}\right)=1$, then there are integers $k_{i}$ such that $\sum k_{i} m_{i}=1$ and then the substitution $g_{i}=t_{n k_{i}}$ results in $w\left(g_{1}, g_{2}, \ldots\right)=t_{\sum n k_{i} m_{i}}=t_{n^{\prime}}$ so every nontrivial word $w$ in which $w^{\prime}$ is not a power is universal for $A(\mathbf{Z})$.

Let $(G, \Omega)$ be a transitive ordered permutation group with congruence $\equiv$. Then the quotient $\Omega / \equiv$ is totally ordered in a natural way and $(G, \Omega)$ induces $(\widehat{G}, \Omega / \equiv)$, where $\widehat{G}=G / L$ with $L$ the lazy subgroup of the action $(G, \Omega /$ $\equiv)$. Henceforth, we will denote $\widehat{G}$ by $G$ and thus make no notational distinction between them. It will be clear from the context which group we are considering. If $\equiv$ and $\approx$ are congruences of $(G, \Omega)$ such that $\approx$ covers $\equiv$ in the sense that $\approx$ contains $\equiv$ and there is no congruence of $(G, \Omega)$ between them, and if $K$ is a $\approx$-class, then $(G, \Omega)$ induces the transitive ordered permutation group $(G, K / \equiv)$, called the component of $(G, \Omega)$ relative to $(\equiv, \approx)$ at $K$ (Lemma 1.7.1 of Glass [4] shows that this component is independent of the particular $\approx$-class chosen $)$, as a subquotient, and $(G, K / \equiv)$ is primitive (Theorem 1.7.2 of Glass [4]). Now the set of all congruences of a transitive ordered permutation group forms a chain under inclusion (Holland [5]). Hence, the set of components is also naturally totally ordered.

Following Adeleke and Holland [1], if $g \in G, \alpha \in \Omega$, and $\alpha g \neq \alpha$, then there is a congruence $\equiv$, maximal with respect to separating $\alpha g$ and $\alpha$. The intersection of all congruences which identify $\alpha g$ and $\alpha$ is a congruence (Corollary $3 \mathrm{~B}$ of Glass [4]) $\approx$ covering $\equiv$, and if $K$ is the $\approx$-class containing $\alpha$, then $K$ contains the nontrivial orbital of $g$ which contains $\alpha$. We refer to the primitive component $(G, K / \equiv)$ as the component determined by $g$ at $\alpha$, and note that it is independent of which point we choose in the orbital of $g$ containing $\alpha$. The ordered set $K$ is isomorphic to the antilexicographically ordered product $\Lambda \overleftarrow{\times} \Gamma$, where $\Lambda$ is any $\equiv$-class and $\Gamma=K / \equiv$.

Now let $\Omega$ be a tree. The next two lemmas give sufficient conditions under which elements of $A(\Omega)$ are conjugate. We will use the following fact to help simplify the notation: Let $\Omega$ be a tree, let $S$ be a nontrivial orbital of some $g \in$ $A(\Omega)$, and let $C \subseteq \Omega$ be a maximal subchain containing $S$. Then $\left.\left(\left.A(\Omega)\right|_{C}\right)\right|_{S}=$ $\left.A(\Omega)\right|_{S}$.

Lemma 2.1 (Generalization of Lemma 5 of Adeleke and Holland [1]). Let $\Omega$ be a tree, let $C \subseteq \Omega$ be a maximal subchain such that $\left(\left.A(\Omega)\right|_{C}, C\right)$ is transitive, 
and for some $g, h \in A(\Omega)$, let $S \subseteq C$ be a nontrivial orbital of both $g$ and $h$ on which $g$ and $h$ have the same parity. Suppose that the maximal primitive component of $\left(\left.A(\Omega)\right|_{S}, S\right)$ is 2-transitive. Then there exists $f \in A(\Omega)$ which maps $S^{c l}$ to itself and fixes $\Omega \backslash S^{c l}$ pointwise, such that $\sigma f^{-1} g f=\sigma h$ for all $\sigma \in S^{c l}$.

Proof. Choose $\alpha \in S$. The sets $\left\{\alpha g^{i}: i \in \mathbf{Z}\right\}$ and $\left\{\alpha h^{i}: i \in \mathbf{Z}\right\}$ are both unbounded above and below in $S$, and $\alpha<\alpha g$ if and only if $\alpha<\alpha h$. Without loss of generality assume that $\alpha<\alpha g$. Now $S$ is isomorphic to $\Lambda \overleftarrow{\times} \Gamma$, where the maximal primitive component $\left(\left.A(\Omega)\right|_{S}, \Gamma\right)$ is 2-transitive. Then from 2-transitivity, the intervals $[\alpha, \alpha g]$ and $[\alpha, \alpha h]$ of $\Gamma$ are isomorphic by an induced element of $\left.A(\Omega)\right|_{S}$. The interval $[\alpha, \alpha g]$ of $S$ is a disjoint union $A \cup\left(\cup B_{\tau}\right) \cup D$, where $A$ is isomorphic to a closed upper segment of $\Lambda, \tau$ ranges over the closed interval $[\alpha, \alpha g]$ of $\Gamma$, each $B_{\tau}$ is isomorphic to $\Lambda$, and $D$ is isomorphic to a closed lower segment of $\Lambda$. Now $\left(\left.A(\Omega)\right|_{S}, S\right)$ is transitive, and thus so is $\left(\left.A(\Omega)\right|_{S}, \Lambda\right)$. Hence all closed upper and lower segments of $\Lambda$ are isomorphic by induced elements of $\left.A(\Omega)\right|_{S}$. Since $[\alpha, \alpha h]$ is constructed in exactly the same way, it follows that there is an induced isomorphism $f_{0}:[\alpha, \alpha g] \rightarrow[\alpha, \alpha h]\left(\left.f_{0} \in A(\Omega)\right|_{S}\right)$. This induces an isomorphism $f_{0}:\langle\alpha, \alpha g\rangle \rightarrow\langle\alpha, \alpha h\rangle$. Now for each $i \in \mathbf{Z}$, let $f_{i}$ be the isomorphism $g^{-i} f_{0} h^{i}$ from $\left\langle\alpha g^{i}, \alpha g^{i+1}\right\rangle$ onto $\left\langle\alpha h^{i}, \alpha h^{i+1}\right\rangle$. By "patching" there exists $f \in A(\Omega)$ which maps $S^{c l}$ onto itself, extends each $f_{i}(i \in \mathbf{Z})$ and fixes $\Omega \backslash S^{c l}$ pointwise. If $\sigma \in S^{c l}$ then $\sigma f^{-1} g f=\sigma f_{i}^{-1} g f_{i+1}=\sigma h$, as required.

Lemma 2.2 (Generalization of Lemma 4 of Adeleke and Holland [1]). Let $\Omega$ be a tree, let $C \subseteq \Omega$ be a maximal subchain such that $\left(\left.A(\Omega)\right|_{C}, C\right)$ is transitive, and for some $g, h \in A(\Omega)$, let $S \subseteq C$ be a nontrivial orbital of both $g$ and $h$. Suppose that the maximal primitive component of $\left(\left.A(\Omega)\right|_{S}, S\right)$ is uniquely transitive. Let $\bar{g}$ denote the induced element of this maximal primitive component. If $\bar{g}=\bar{h}$ then there exists $f \in A(\Omega)$ which maps $S^{c l}$ onto itself and fixes $\Omega \backslash S^{c l}$ pointwise, such that $\sigma f^{-1} g f=\sigma h$ for all $\sigma \in S^{c l}$.

Proof. Choose $\alpha \in S$. The sets $\left\{\alpha g^{i}: i \in \mathbf{Z}\right\}$ and $\left\{\alpha h^{i}: i \in \mathbf{Z}\right\}$ are both unbounded above and below in $S$, and $\alpha<\alpha g$ if and only if $\alpha<\alpha h$. Without loss of generality assume that $\alpha<\alpha g$. Now $S$ is isomorphic to $\Lambda \overleftarrow{\times} \Gamma$, where the maximal primitive component $\left(\left.A(\Omega)\right|_{S}, \Gamma\right)$ is uniquely transitive. The interval $[\alpha, \alpha g]$ of $S$ is a disjoint union $A \cup\left(\cup B_{\tau}\right) \cup D$, where $A$ is isomorphic to a closed upper segment of $\Lambda, \tau$ ranges over the closed interval $[\alpha, \alpha g]=[\alpha, \alpha h]$ of $\Gamma$, each $B_{\tau}$ is isomorphic to $\Lambda$, and $D$ is isomorphic to a closed lower segment of $\Lambda$. The proof then follows as in the proof of Lemma 2.1.

Let $\Omega$ be a tree. Following Droste, Holland, and Macpherson [3], we let $\operatorname{ram}(\Omega):=\{\alpha \in \bar{\Omega}: \alpha=\inf \{\tau, \sigma\}$ for some $\tau, \sigma \in \Omega$ with $\tau \| \sigma\}$, the set of ramification points of $\Omega$. Let $\Omega$ be a tree and $\alpha \in \operatorname{ram}(\Omega)$. The maximal 
lower directed subsets of $\{\tau \in \Omega: \alpha<\tau\}$ are called the cones of $\alpha$. We let $C(\alpha)$ denote the set of all cones of $\alpha$. Let $\kappa$ be a finite or infinite cardinal. If $|C(\alpha)|=\kappa$ for all $\alpha \in \operatorname{ram}(\Omega), \kappa$ is called the ramification order of $\Omega$, and we write $\kappa=$ r.o. $(\Omega)$.

Theorem 2.3. Let $\Omega$ be a tree. If $\Omega$ has at least $n\left(1<n \in \mathbf{Z}^{+}\right)$pairwise isomorphic cones at some $\alpha \in \operatorname{ram}(\Omega)$, then the word $x^{n}$ is not universal for $A(\Omega)$.

Proof. Choose $A_{i} \in C(\alpha) \quad(i=1, \ldots, n)$ such that $\left\{A_{i}\right\}$ is a collection of pairwise isomorphic cones. Then there exist isomorphisms $h_{i}: A_{i} \rightarrow A_{i+1}$ $(i=1, \ldots, n-1)$. Define $g \in A(\Omega)$ by

$$
g= \begin{cases}h_{i} \text { on } A_{i} & (i=1, \ldots, n-1) \\ \left(h_{1} h_{2} \cdots h_{n-1}\right)^{-1} & \text { on } A_{n} \\ e \text { on } \Omega \backslash \bigcup\left\{A_{i}\right\} & \end{cases}
$$

Then $g^{n}=e$ and $\operatorname{supp}(g)=\bigcup\left\{A_{i}\right\}$. Now suppose there exists $f \in A(\Omega)$ such that $f^{n}=g$. We need the following three lemmas.

Lemma 2.4. If $\tau \in \bigcup\left\{A_{i}\right\}$, then also $\tau f \in \bigcup\left\{A_{i}\right\}$.

Proof. Suppose $\tau f \notin \bigcup\left\{A_{i}\right\}$. Then $(\tau f) g=\tau f \Rightarrow(\tau f) f^{n}=\tau f \Rightarrow \tau f^{n}=\tau \Rightarrow$ $\tau g=\tau$, a contradiction.

Lemma 2.5. If $\tau \in \operatorname{supp}(f)$, then $\tau f \| \tau$.

Proof. Suppose $\tau f>\tau$. Then $\tau<\tau f<\tau f^{2}<\cdots<\tau f^{n^{2}}=\tau\left(f^{n}\right)^{n}=\tau g^{n}=$ $\tau e=\tau$, a contradiction. Similarly, if $\tau f<\tau$.

Lemma 2.6. $\alpha f=\alpha$.

Proof. Suppose $\alpha f \neq \alpha$. Then from Lemma 2.5 we have $\alpha f \| \alpha$. Let $\tau \in A_{1}$. Then $\alpha<\tau$ and so $\alpha f<\tau f$. Now from Lemma 2.4, $\tau f \in \bigcup\left\{A_{i}\right\}$ and so $\alpha<\tau f$. Since $\{\sigma \in \Omega: \sigma<\tau f\}$ is a chain we have either $\alpha<\alpha f$ or $\alpha f<\alpha$, a contradiction.

Now from Lemmas 2.4 and 2.6, we see that $f$ permutes the set $\left\{A_{i}\right\}$, and thus $f$ induces a solution to the equation $x^{n}=(12 \ldots n)$ in $S_{n}$ (the symmetric group on $n$ symbols), a contradiction since it is well known that the equation $x^{n}$ equal to an $n$-cycle $\left(1<n \in \mathbf{Z}^{+}\right)$has no solution in any symmetric group.

Corollary 2.7. Let $\Omega$ be a tree. If $\Omega$ has infinitely many pairwise isomorphic cones at some $\alpha \in \operatorname{ram}(\Omega)$, then every free group word $w$, which is a proper power, is not universal for $A(\Omega)$.

A partially ordered permutation group is a pair $(G, \Omega)$, where $\Omega$ is a p.o. set and $G$ is a subgroup of the order-automorphism group $A(\Omega)$. We now define the wreath product of two partially ordered permutation groups. The following lemma is easily verified, where we write $\left\{g_{\tau}\right\}$ as a shorthand expression for $\left\{g_{\tau}: \tau \in T\right\}$. 
Lemma 2.8 (Modification of Lemma 5.1 of Glass [4]). Let $(G, \Omega)$ and $(H, T)$ be partially ordered permutation groups. Let $\Lambda=\Omega \times T$ and $W=\left\{\left(\left\{g_{\tau}\right\}, h\right)\right.$ : $h \in H$ and $g_{\tau} \in G$ for all $\left.\tau \in T\right\}$. Define a binary operation on $W$ by: $\left(\left\{f_{\tau}\right\}, h_{1}\right)\left(\left\{g_{\tau}\right\}, h_{2}\right)=\left(\left\{a_{\tau}\right\}, h_{1} h_{2}\right)$, where $a_{\tau}=f_{\tau} g_{\tau h_{1}}(\tau \in T)$. Then $W$ is a group with identity $\left(\left\{e_{\tau}\right\}, e\right)$, where $e_{\tau}=e(\tau \in T)$, and $\left(\left\{g_{\tau}\right\}, h\right)^{-1}=$ $\left(\left\{f_{\tau}\right\}, h^{-1}\right)$, where $f_{\tau}=\left(g_{\tau h^{-1}}\right)^{-1}(\tau \in T)$; and $(W, \Lambda)$ is a partially ordered permutation group if $(\alpha, \sigma)\left(\left\{g_{\tau}\right\}, h\right):=\left(\alpha g_{\sigma}, \sigma h\right)(\alpha \in \Omega, \sigma \in T)$.

$(W, \Lambda)$ is called the Wreath product of $(G, \Omega)$ and $(H, T)$, and is written $(G, \Omega) W r(H, T)$. If $f=\left(\left\{g_{\tau}\right\}, h\right) \in W$, then $h$ is called the global component of $f$ and $\left\{g_{\tau}\right\}$ the set of local components of $f$. We will also write $W=G W r H$ if we wish to emphasize the group part of $(G, \Omega) W r(H, T)$.

Now let $\Omega$ be a tree with infinitely many pairwise isomorphic cones at some $\alpha \in \operatorname{ram}(\Omega)$. Choose $B_{i} \in C(\alpha)\left(i \in \mathbf{Z}^{+}\right)$such that $\left\{B_{i}\right\}$ is a collection of pairwise isomorphic cones, and let $\Gamma=\bigcup\left\{B_{i}\right\}$. Let $w$ be any nontrivial free group word and let $g \in A(\Omega)$ be such that $\operatorname{supp}(g) \subseteq \Gamma$. Then solving the equation $w=g$ in $A(\Omega)$, with elements whose supports are contained in $\Gamma$, is equivalent to solving the equation $w=\bar{g}$ (where $\bar{g}$ is the element of $A(\Gamma)$ induced by $g)$ in $(A(\Gamma), \Gamma)=\left(A\left(B_{1}\right), B_{1}\right) W r\left(S\left(\mathbf{Z}^{+}\right), \mathbf{Z}^{+}\right)$, where we view $\mathbf{Z}^{+}$ as a totally unordered set. This suggests the following more general problem.

Let $(G, \Omega)$ be any partially ordered permutation group. Which nontrivial free group words are universal for $(G, \Omega) \operatorname{Wr}(S(V), V)$, where $V$ is any trivially ordered countably infinite set? Clearly proper powers are not since $S(V)$ is a homomorphic image of $G W r S(V)$. What about other words? We now consider an example.

Let $w=x^{2} y^{2}$ and let $g=\left(\left\{g_{\tau}\right\}, h\right) \in G W r S(V)$. Suppose there exist $a=\left(\left\{a_{\tau}\right\}, h_{1}\right), b=\left(\left\{b_{\tau}\right\}, h_{2}\right) \in G W r S(V)$ such that $a^{2} b^{2}=g$. Then for each $(\alpha, \sigma) \in \Omega \overleftarrow{\times} V$ we have $(\alpha, \sigma) a^{2} b^{2}=(\alpha, \sigma) g$, and carrying out the algebra we see that $\alpha a_{\sigma} a_{\sigma h_{1}} b_{\sigma h_{1}} b_{\sigma h_{1}^{2} h_{2}^{2}}=\alpha g_{\sigma}$ and $\sigma h_{1}^{2} h_{2}^{2}=\sigma h$. This indicates that a solution (if any) of the general equation $w=g, w$ a nontrivial free group word which is not a proper power and $g=\left(\left\{g_{\tau}\right\}, h\right) \in G W r S(V)$, is dependent on how the global solution to the equation $w=h$ is constructed in $S(V)$. From Lyndon [6] and Mycielski [9] we know that we can solve the equation $w=h$ in $S(V)$. The construction involves graph theory.

We now give some background information concerning graphs. Following Lyndon [6], by a graph $\Gamma$ we mean a 1-complex; to each edge $e$ there corresponds an inverse edge $e^{-1}$ with $\left(e^{-1}\right)^{-1}=e$ and $e^{-1} \neq e$. We consider only labelled graphs: Let $B$ be a basis for some free group $F$ and let $X=B \cup B^{-1}$. A function $\Phi$ is given assigning to each edge $e$ its label $\Phi(e)=x$ in $X$, with $\Phi\left(e^{-1}\right)=x^{-1}$. The label on a path $\alpha=e_{1} \cdots e_{n}$ is defined to be $\Phi(\alpha)=\Phi\left(e_{1}\right) \ldots \Phi\left(e_{n}\right)$. If for each vertex $P$ and each $x$ in $X$, there is exactly one edge at $P$ with label $x$, then the graph $\Gamma$ is a total graph. 
The concept of a total graph with vertex set $V$ is equivalent to that of a homomorphism from $F$ into $S(V)$. Whenever a total graph $\Gamma$ is mentioned with a word $w$, we assume that $\Gamma$ is a total graph with respect to the variables appearing in $w$.

Definition 2.9. A $w$-path of a total graph $\Gamma$ is a path with label $w$. A $w$-path is said to be completed when each of its edges has been marked. We leave as undefined what it means for an edge to be marked.

Now let $(G, \Omega)$ be a partially ordered permutation group, let $w$ be a nontrivial free group word which is not a proper power, and let $g=\left(\left\{f_{\tau}\right\}, h\right) \in$ $(G, \Omega) W r(S(V), V)$, where $V$ is any trivially ordered countably infinite set. Then the equation $w=g$ has a solution in $G W r S(V)$ if there exists a total graph $\Gamma$ which (1) for $w$, represents the cycle structure of $h$, and for which (2) there exists an algorithm to mark all the edges of $\Gamma$ such that whenever an edge is marked at most one $w$-path is completed. The result of Lyndon [6] and Mycielski [9] gives the existence of a total graph $\Gamma$ satisfying (1). Condition (2) would allow us to correctly choose the local components of a solution without having to solve simultaneous systems of equations. This suggests the following strictly graph theoretic question: let $V$ be any countably infinite set. For each pair $(w, h)$, where $w$ is a nontrivial free group word which is not a proper power and $h \in S(V)$, does there exist a total graph $\Gamma$ satisfying both (1) and (2)? In particular, do the total graphs constructed in Lyndon [6] satisfy (2)?

Lemma 2.10. Let $(G, \Omega)$ be a partially ordered permutation group, and let $(W, \Lambda)=(G, \Omega) W r(S(V), V)$, where $V$ is any trivially ordered countably infinite set. Let $w$ be a nontrivial free group word which is not a proper power, and let $g=\left(\left\{f_{\tau}\right\}, h\right) \in W$ be such that $h$ has one infinite orbit consisting entirely of $V$. Then the equation $w=g$ has a solution in $W$.

Proof. From Lyndon [6] and Mycielski [9] we can solve $w=h$ in $S(V)$, and so there exist $h_{1}, h_{2}, \ldots \in S(V)$ such that $w\left(h_{1}, h_{2}, \ldots\right)=h$. Now let $g_{i}=\left(\left\{k_{i, \tau}\right\}, h_{i}\right) \in W$, where $k_{i, \tau}$ is defined as follows: let $n_{i, \tau}$ be the unique positive integer such that $\tau h^{n_{i, \tau}}=\tau h_{i}$. We define $k_{i, \tau}$ by $\alpha k_{i, \tau}=\mu$, where $\left(\mu, \tau h^{n_{i, \tau}}\right)=(\alpha, \tau) g^{n_{i, \tau}}(\alpha \in \Omega)$. Then $w\left(g_{1}, g_{2}, \ldots\right)=g$.

The following lemma is due to Adeleke and Holland [1987].

Lemma 2.11 (Lemma 2 of Adeleke and Holland [1]). If $\Omega$ is a 2-transitive chain which has a countable subset with no upper bounds and no lower bounds, and $w$ is any nontrivial free group word, then there is a substitution in $A(\Omega)$ such that $w\left(g_{1}, g_{2}, \ldots\right)$ has only one orbital and moves every point of $\Omega$ up.

Proof. By hypothesis, we may find a set $\{\sigma(i): i \in \mathbf{Z}\} \subseteq \Omega$ which has no upper or lower bounds. Let $x_{1}, x_{2}, \ldots, x_{k}$ be the variables involved in the reduced nontrivial word $w$, and let $w=z_{1} z_{2} \ldots z_{j}$ where for each $n, z_{n} \in$ $\left\{x_{1}, x_{1}^{-1}, x_{2}, x_{2}^{-1}, \ldots, x_{k}, x_{k}^{-1}\right\}$. Our goal is to find substitutions $g_{r} \in A(\Omega)$ 
such that for each $i, \sigma(i) w\left(g_{1}, g_{2}, \ldots\right)=\sigma(i+1)$. Clearly, 2-transitivity implies that the order of $\Omega$ is dense. Choose points $\tau(i, s), i \in \mathbf{Z}, s=$ $1,2, \ldots, j-1$, such that for each $i, \sigma(i)<\tau(i, 1)<\tau(i, 2)<\ldots<\tau(i, j-1)$ $<\sigma(i+1)$. Since $z_{1}$ is $x_{r}$ or $x_{r}^{-1}$ for some $r$, 2-transitivity together with "patching" implies that we may construct $g_{r} \in A(\Omega)$ such that for each $i$, $\sigma(i) z_{1}=\tau(i, 1)$. Similarly, we may arrange that for each $n=2, \ldots, j-1$, $\tau(i, n-1) z_{n}=\tau(i, n)$, and that $\tau(i, j-1) z_{j}=\sigma(i+1)$. The result is that for each $i, \sigma(i) w\left(g_{1}, g_{2}, \ldots\right)=\sigma(i+1)$. Hence, $w\left(g_{1}, g_{2}, \ldots\right)$ has just one orbital, consisting of all of $\Omega$. Furthermore, if we prefer we may choose our substitution such that $w\left(g_{1}, g_{2}, \ldots\right)$ has only one orbital and moves every point of $\Omega$ down.

We say that a tree $\Omega$ is chain transitive if for each maximal subchain $C \subseteq \Omega$, $\left(\left.A(\Omega)\right|_{C}, C\right)$ is a transitive ordered permutation group (i.e., for each $\alpha, \beta \in C$ there exists $g \in A(\Omega)$ such that $\alpha g=\beta$ and $C g=C)$.

Lemma 2.12. Let $\Omega$ be a chain-transitive tree such that for each maximal subchain $C \subseteq \Omega$, no primitive component of $\left(\left.A(\Omega)\right|_{C}, C\right)$ is uniquely transitive. Let $w$ be a nontrivial free group word, and let $S$ be a nontrivial orbital of some $g \in A(\Omega)$. Then there is a substitution in $A(\Omega)$ such that $w\left(g_{1}, g_{2}, \ldots\right)=g$ on $S^{c l}$ and $\operatorname{supp}\left(g_{i}\right) \subseteq S^{c l}$.

Proof. Let $C \subseteq \Omega$ be any maximal subchain containing $S$. Now no primitive component of $\left(\left.A(\Omega)\right|_{S}, S\right)\left(=\left(\left.\left(\left.A(\Omega)\right|_{C}\right)\right|_{S}, S\right)\right)$ is uniquely transitive. Moreover, $\left(\left.A(\Omega)\right|_{S}, S\right)$ has a maximal primitive component, and hence this maximal primitive component must be 2-transitive (Theorem 1.2, Lemma 3.13 of Maroli [7]). Without loss of generality assume that $g$ has positive parity on $S$. Now since $\left(\left.A(\Omega)\right|_{S}, S\right)$ is closed under "patching" (Lemma 3.13 of Maroli [7]), it follows as in the proof of Lemma 2.11 that there are substitutions $\left.g_{i} \in A(\Omega)\right|_{S}$ such that $w\left(g_{1}, g_{2}, \ldots\right)$ has only one positive orbital and moves every point of $S$ up. It follows that the member of the maximal component induced by $w\left(g_{1}, g_{2}, \ldots\right)$ also has only one positive orbital and moves every point up. Extend each $g_{i}$ to an element of $A(\Omega)$ such that $\operatorname{supp}\left(g_{i}\right) \subseteq S^{c l}$. Then from Lemma 2.1, there exists $f \in A(\Omega)$ such that $f^{-1} w\left(g_{1}, g_{2}, \ldots\right) f=g$ on $S^{c l}$ and $\operatorname{supp}(f) \subseteq S^{c l}$. Let $h_{i}=f^{-1} g_{i} f$. Then $w\left(h_{1}, h_{2}, \ldots\right)=g$ on $S^{c l}$ and $\operatorname{supp}\left(h_{i}\right) \subseteq S^{c l}$.

Let $\Omega$ be a tree. For notational purposes we define $G_{1}(\Omega):=\{g \in A(\Omega)$ : whenever there exists $\alpha \in \operatorname{ram}(\Omega)$ with $\alpha g=\alpha$, then for all $B \in C(\alpha)$, $B g=B$ (i.e., $g$ acts like the identity on $C(\alpha))\}$; and $G_{2}(\Omega):=\{g \in A(\Omega)$ : whenever there exists $\alpha \in \operatorname{ram}(\Omega)$ with $\alpha g=\alpha$, then for all $B \in C(\alpha)$, either $B g=B$ or $|B\langle g\rangle|=\aleph_{0}$ (i.e., all the nontrivial orbits of $g$ acting on $C(\alpha)$ are infinite) $\}$.

Theorem 2.13. Let $\Omega$ be a chain-transitive tree such that for each maximal subchain $C \subseteq \Omega$, no primitive component of $\left(\left.A(\Omega)\right|_{C}, C\right)$ is uniquely transitive. 
Then if (i) $w$ a nontrivial free group word and $g \in G_{1}(\Omega)$, or (ii) $w$ is a nontrivial free group word which is not a proper power and $g \in G_{2}(\Omega)$, we can solve the equation $w=g$ in $A(\Omega)$.

Proof. (i) Now $\operatorname{supp}(g)=\bigcup\left\{S^{c l}: S\right.$ is a nontrivial orbital of $\left.g\right\}$, and this union is disjoint. We will construct our substitutions separately on each $S^{c l}$. Let $S$ be a nontrivial orbital of $g$. From Lemma 2.12, there is a substitution $g_{i} \in A(\Omega)$ with $w\left(g_{1}, g_{2}, \ldots\right)=g$ on $S^{c l}$ and $\operatorname{supp}\left(g_{i}\right) \subseteq S^{c l}$. We do this on each $S^{c l}, S$ a nontrivial orbital of $g$, and then patch together the resulting substitutions.

(ii) Now $\operatorname{supp}(g)=\bigcup\left\{S^{c l}: S\right.$ a nontrivial orbital of $\left.g\right\} \cup \bigcup\{B\langle g\rangle$ : there exists $\alpha \in \operatorname{ram}(\Omega)$ and $B \in C(\alpha)$ such that $\alpha g=\alpha$ and $B g \neq B\}$. The set $B\langle g\rangle$ will be referred to as a nontrivial cone orbital of $g$. We will construct our substitutions separately on each $S^{c l}$ and $B\langle g\rangle$. Let $S$ be a nontrivial orbital of $g$. From Lemma 2.12, there is a substitution $g_{i} \in A(\Omega)$ with $w\left(g_{1}, g_{2}, \ldots\right)=$ $g$ on $S^{c l}$ and $\operatorname{supp}\left(g_{i}\right) \subseteq S^{c l}$. Now let $B\langle g\rangle$ be an arbitrary nontrivial cone orbital of $g$. Note that $B\langle g\rangle$ is a partially ordered set which is not a tree, and that $(A(B\langle g\rangle), B\langle g\rangle) \cong(A(B), B) W r(S(V), V)$, where $V$ is a trivially ordered countably infinite set. Thus from Lemma 2.10 , there is a substitution $g_{i} \in A(B\langle g\rangle)$ with $w\left(g_{1}, g_{2}, \ldots\right)=\left.g\right|_{B\langle g\rangle}$. Now extend each $g_{i}$ to an element of $A(\Omega)$ such that $\operatorname{supp}\left(g_{i}\right) \subseteq B\langle g\rangle$. We do this on each $S^{c l}, S$ a nontrivial orbital of $g$, and on each nontrivial cone orbital of $g$, and then patch together the resulting substitutions.

Corollary 2.14 (Theorem 7 of Adeleke and Holland [1]). Let $\Omega$ be a transitive chain and suppose $\Omega$ has no primitive component which is uniquely transitive. Then every nontrivial free group word $w$ is universal for $A(\Omega)$.

Proof. If $\Omega$ is a chain, then $G_{1}(\Omega)$ is equal to $A(\Omega)$.

Corollary 2.15. Let $\Omega$ be a 2-transitive strict tree which is not 2-homogeneous. Then every nontrivial free group word $w$ is universal for $A(\Omega)$.

Proof. From Proposition 4.2 of Droste, Holland, and Macpherson [3] we have for each maximal subchain $C \subseteq \Omega,\left(\left.A(\Omega)\right|_{C}, C\right)$ is 2-transitive and thus primitive (Corollary 1.6.6 of Glass [4]). Hence $\Omega$ satisfies the conditions of Theorem 2.13. Now a 2-transitive strict tree is a tree in the sense of Droste [2] (Definition 5.1), and thus from Theorem 5.17 and Corollaries 5.36 and 5.37 of Droste [2], we see that r.o. $(\Omega)=2$ and $\Omega$ has two nonisomorphic cones at each ramification point. Therefore, $G_{1}(\Omega)$ is equal to $A(\Omega)$.

Theorem 2.16. If $\Omega$ is a chain-transitive tree and $w$ is a nontrivial free group word such that $w^{\prime}$ ( the abelianized form of $w$ ) is not a power and $g \in G_{2}(\Omega)$, then we can solve the equation $w=g$ in $A(\Omega)$.

Proof. We proceed exactly as in the proof of Theorem 2.13 (ii), except that we may encounter nontrivial orbitals $S$ of $g$ such that $\left(\left.A(\Omega)\right|_{S}, S\right)$ has a maximal 
component which is uniquely transitive. In this case we proceed as follows: let $\left(\left.A(\Omega)\right|_{S}, K\right)$ denote this maximal primitive component, which we may consider to be a subgroup of the reals, acting on itself by translation. Let $\bar{g}$ denote the induced element of this maximal component, and let $t_{r}$ denote translation by $\bar{g}(r \in \mathbf{R})$. Let $w^{\prime}=\prod x_{i}^{m_{i}}$. Since $w^{\prime}$ is not a power, g.c.d. $\left(m_{i}\right)=1$, and thus there are integers $k_{i}$ such that $\sum k_{i} m_{i}=1$. Then the substitution $\overline{g_{i}}=t_{r k_{i}}$ results in $w\left(\overline{g_{1}}, \overline{g_{2}}, \ldots\right)=t_{\sum r k_{i} m_{i}}=t_{r}=\bar{g}$. Extend each $\overline{g_{i}}$ arbitrarily to an element $g_{i}$ of $A(\Omega)$ such that $\operatorname{supp}\left(g_{i}\right) \subseteq S^{c l}$. Then $w\left(g_{1}, g_{2}, \ldots\right)$ induces $\bar{g}$ on $\left(\left.A(\Omega)\right|_{S}, K\right)$. Now from Lemma 2.2 , there exists $f \in A(\Omega)$ such that $f^{-1} w\left(g_{1}, g_{2}, \ldots\right) f=g$ on $S^{c l}$ and $\operatorname{supp}(f) \subseteq S^{c l}$. Let $h_{i}=f^{-1} g_{i} f$. Then $w\left(h_{1}, h_{2}, \ldots\right)=g$ on $S^{c l}$ and $\operatorname{supp}\left(h_{i}\right) \subseteq S^{c l}$.

Corollary 2.17 (Theorem 8 of Adeleke and Holland [1]). If $\Omega$ is a transitive chain and $w$ is a nontrivial free group word such that $w^{\prime}$ is not a power, then $w$ is universal for $A(\Omega)$.

Proof. If $\Omega$ is a chain, then $G_{2}(\Omega)$ is equal to $A(\Omega)$.

\section{ACKNOWLEDGMENT}

This is a polished version of a chapter of the author's Ph.D. dissertation written at Bowling Green State University. The author wishes to express his appreciation to Professor W. Charles Holland for his encouragement and guidance in the completion of this work.

\section{BIBLIOGRAPHY}

1. S. A. Adeleke and W. C. Holland, Representation of order automorphisms by words, preprint, 1987.

2. M. Droste, Structure of partially ordered sets with transitive automorphism groups, Mem. Amer. Math. Soc., no. 334, Amer. Math. Soc., Providence, RI, 1985.

3. M. Droste, W. C. Holland, and H. D. Macpherson, Automorphism groups of infinite semilinear orders (I), Proc. London Math. Soc. 58 (1989), 454-478.

4. A. M. W. Glass, Ordered permutation groups, London Math. Soc. Lecture Note Ser. $\mathbf{5 5}$ (1981).

5. W. C. Holland, Transitive lattice-ordered permutation groups, Math. Z. 87 (1965), 420-433.

6. R. C. Lyndon, Words and infinite permutations, preprint, 1986.

7. J. A. Maroli, Tree permutation groups, Doctoral Dissertation, Bowling Green State University, 1989.

8. S. H. McCleary, o-primitive ordered permutation groups, Pacific J. Math. 40 (1972), 349372.

9. J. Mycielski, Representations of infinite permutations by words, Proc. Amer. Math. Soc. $\mathbf{1 0 0}$ (1987), 237-241.

10. D. M. Silberger, Are primitive words universal for infinite symmetric groups?. Trans. Amer. Math. Soc. 276 (1983), 841-852.

Department of Mathematical Sciences, Ohio Wesleyan University, Delaware, Ohio 43015 